\title{
Predictive value of urgent referrals for suspected oral cancer
}

The two-week wait cancer initiative on oral cancer; the predictive value of urgent referrals to an oral medicine unit P. Singh and S. Warnakulasuriya Br Dent J 2006; 201: 717-720

\section{Background}

The two-week wait cancer initiative was designed to speed up referral of patients suspected of having cancer. The National Institute of Clinical Excellence (NICE) have issued guidelines for head and neck cancers warranting urgent referral.

\section{Objective}

To look at the appropriate use of the two-week wait/urgent referrals by measuring the proportion of urgent referrals found to have cancer, to assess the sensitivity of the clinical guidelines and to explore how practitioners used them in primary care.

\section{Method}

Collection and analysis of urgent referral letters and the clinic outcome for urgently referred cases over a one-year period in an oral medicine department. Screening of the biopsy service database to reveal any routine referrals subsequently found to have cancer.

Results

None of the routine referrals but eight percent of all urgent patients were found to have cancer, equating to $24 \%$ of the group in which malignancy was suspected. The predictive value of referring a case as suspected of oral cancer/with cancer symptoms was low, estimated at $7.9 \%$.

\section{Conclusion}

The referral guidelines on oral cancer symptoms developed by NICE may need further revision. Moreover, development of other appropriate adjuncts that aid visual inspection for the detection of oral cancer may improve the sensitivity of positive detections in primary care.

\section{IN BRIEF}

- Among urgent referrals to an oral medicine unit over a one year period, six were found to have oral cancer.

- The predictive value of referring a suspected cancer marked 'urgent' was estimated to be $8 \%$.

- Among routine referrals, none were diagnosed with cancer.

- Greatest delay in oral cancer diagnosis remains patients seeking advice from a primary care dentist or a physician but with rapid access for cancer further delays could be reduced to a minimum.

- UK guidelines to identify and refer patients with head and neck cancer are available but in light of the findings from this study may need further clarification or revision.

\section{COMMENT}

The recent NICE guidelines dictate that all cases of suspected oral cancer should be seen by a specialist within two weeks of referral. Early diagnosis offers the patient the best possible chance of curative treatment and 'fast tracking' patients with suspected oral cancer is the objective of the guidelines. As with any service improvement initiative, it is essential that the outcome is evaluated, particularly so in this case as there does not appear to have been a pilot study prior to implementation. This is therefore a timely and appropriate paper.

Few if any diagnostic pathways are completely secure and oral cancer is no exception. Problems stem from the low incidence of the disease and the fact that the clinical symptoms and signs - ulceration, white and red lesions and swellings - are common to many other innocent diagnoses such as traumatic ulcers, frictional keratosis, candidosis and lichen planus. To be effective the guidelines need to be sensitive enough to distinguish innocent from malignant.

The study shows that the sensitivity of the guidelines, ie the number of cancers found in the urgent referral group, was $8 \%$. Notably, all these came from the 25 referrals where malignancy was suspected with all other urgent referrals proving to be innocent. Perhaps of more significance is the obverse, the specificity of the test. This is the probability that a case referred as non urgent does not have cancer. The study addresses this by looking at all oral cancers found from the total number of referrals. Significantly, no cancers were found in the 694 patients referred as routine, a specificity of $100 \%$.

Although this high specificity is reassuring, it is disappointing to see that cases graded urgent solely on descriptive features did not yield any positive cases. This suggests that the clinical signs that underpin the guidelines are not sensitive enough to grade patients as urgent. Ulceration is a good example, with the guidelines making no reference to key signs such as raised rolled edge and induration. Also, as referenced, this type of approach may impact adversely on waiting times and the diagnostic and treatment pathways.

Whereas prevention remains the key to disease control, improving patient awareness coupled with appropriate and timely treatment are clearly essential. The UK is unique in attempting to define a minimum period to commence the latter. This paper would indicate that the current guidelines are not yet sensitive enough to facilitate this and should be considered for review.

C. G. Cowan, Consultant Oral Surgeon, Royal Group of Hospitals/ Head of Division, Oral Surgery, Oral Medicine \&t Oral Pathology, Queen's University Belfast

DOI: $10.1038 /$ sj.bdj.4814306 